\title{
Multi-Dose Container for Nasal and Ophthalmic Drugs: A Preservative Free Future?
}

\author{
Degenhard Marx and Matthias Birkhoff \\ Aptar Pharma, Consumer Healthcare Division, \\ Germany
}

\section{Introduction}

The first nasal spray pumps were developed some 50 years ago and replaced step by step droppers and pipettes. Now they are widely used to moisturize the nasal mucosa using saline solutions (from a regulatory point of view considered medical devices under the EU directive) or as nasal preparations for the administration of topically acting drugs (e.g. nasal decongestants or nasal steroids) or for the non-invasive administration of substances which need to reach systemic circulation (anti-migraine medication, hormones). For chronic diseases like allergic rhinitis, multi-dose devices are very cost effective and convenient and provide the safety and precision regulatory bodies require. To date, most of the medications administered nasally contain a preservative to support long storage times and proper in-use stability.

For ophthalmic drugs, there was a similar development from simple droppers to more sophisticated devices. Squeeze bottles without metering function are still widely used for ophthalmic medications especially for chronic conditions. Artificial tears filled in multi-dose bottles are commonly used to relieve dry eye symptoms, these again being medical devices because the mode of action is purely physical. Looking at active drugs, the most successful in the ophthalmic field are drugs (e.g. beta-antagonists and prostaglandine-analogues) for the treatment of glaucoma and anti-histamines to treat allergic conjunctivitis.

The reader may wonder why nasal spray pumps together with multi-dose ophthalmic droppers are considered in the very same chapter. The answer is that both device classes share the same blueprint: they use a bottle made of plastic or glass attached to a pump or dropper which generates and delivers a more or less metered dose and on top of this serves as a closure. So the pumps or droppers support two general functions:

- Sealing of the container and protection of the content during storage and transportation.

- Delivery of a metered dose of liquid or viscous pharmaceutical formulation.

The major challenge during development is to reach a reliable delivery of the metered dose without having any negative impact caused by drug-device interactions.

\section{Intranasal administration}

Intranasal administration has a long tradition. Around three thousand years ago intranasal application of dried scabs was used to vaccinate against smallpox in India. Later intranasal administration of psychotropic drugs was and still is used for medical as well as recreational 
purposes. Leaving these ancient therapeutic regimes behind, today the topical treatment of allergic rhinitis using anti-histamines (azelastine, levocabastine, olopatadine) or glucocorticoids (e.g. budesonide, fluticasone or ciclesonide) is well established. For this particular indication, drugs should act fast and just locally while systemic absorption should be as low as possible to avoid systemic side effects which are linked with typical oral formulations of comparable drug substances. Since allergic rhinitis is a chronic disease, only multi-dose bottles are used there.

\section{Nasal decongestants}

e.g. oxymetazoline, xylometazoline, naphazoline, tetryzoline

\section{Intranasal steroids}

e.g. beclomethasone, budesonide,

fluticasone, mometasone, ciclesonide

\section{Antihistamines}

azelastine, levocabastine, olopatadine

\section{Triptanes}

sumatriptan, zolmitriptan

\section{Analgesics}

fentanyl, ketorolac, (ketamine)

\section{Preferred profile}

Local activity only, low systemic adsorption to minimize side effects (headache and dizziness)

Local activity only, low systemic adsorption to minimize systemic side effects (cortisol suppression)

Local activity only, low systemic adsorption to minimize systemic side effects (sedation)

Fast onset of action, high systemic adsorption to blunt migraine attacks

Fast onset of action but avoid high plasma concentration peaks

Table 1. Established drug classes for intranasal administration

But intranasal administration has much more potential. The nasal mucosa can be used for non-invasive systemic administration of drugs. The nasal mucosa in humans is around $150 \mathrm{~cm}^{2}$, a surface which is well supplied by blood vessels. This ensures a rapid absorption of most drugs and can generate high blood levels. This administration route avoids the first pass metabolism which needs to be taken into account following oral administration. This bypassing of the gastric system even enables the delivery of peptide hormones. Calcitonin and desmopressin are on the market for years now, insulin and glucagon under clinical development for this administration route (Leary et al., 2008).

Intranasal administration is considered a non-invasive administration route and easy to do for self-administration or for care-givers with low potential for injuries or disease transmission (hepatitis B, HIV). This is of special importance if fast pain relief is required and patient's ability to deal with injections is impaired. Intranasal triptanes for migraine treatment, fentanyl to stop breakthrough pain and ondansetrone to relieve nausea take advantage of this administration route. For these indications, single dose devices or multidose devices with counting or lock-out mechanisms are available to reduce the risk of unintended overdosing or misuse.

A controversial discussion is ongoing regarding direct nose to brain drug delivery. This would open the door to a targeted treatment for various CNS diseases like Alzheimer, Parkinson, and epilepsies. Unfortunately, the available literature does not suggest consistent findings, more research is required here (Wen, 2011). 


$\begin{array}{llc} & \text { Indication } & \text { Bioavailability [\%] } \\ \text { Azelastine } & \text { Topical anti-histamine } & 40 \\ \text { Budesonide } & \text { Topical glucocorticoid } & 34 \\ \text { Fentanyl } & \text { Opiate analgesic } & 80-90 \\ \text { Sumatriptan } & \text { Anti-migraine } & 16 \\ \text { Calcitonin } & \text { Bone mineralization, polypeptide (32 amino acids) } & \sim 3 \\ \text { Desmopressin } & \text { Antidiuretic and anti-heamophilic, cyclic } & 10-20 \\ & \text { nonapeptide } & 12-15 \%\end{array}$

Table 2. Nasal bioavailability of selected drugs (marketed or in late stage development)

For the successful development of a nasal spray it is important to understand the basic mechanisms of such a device to recognize the cliffs which must be avoided. Of course a product intended to be administered as a nasal spray should have no unpleasant smell, should not be irritating and even long term treatment should have no negative effects on the nasal mucosa (e.g. ulceration, loss of sense of smell). There should be also no safety concern, if a dose is unintentionally shot into the eyes.

For most pumps the dispensed volume per actuation is set between 50 and $140 \mu \mathrm{l}$, and an administered volume of $100 \mu \mathrm{l}$ per nostril is optimum in adults. Higher volumes are prone to drip out immediately or to flow down into the throat (post nasal drip) and subsequent swallowing. So the anticipated dose should fit into a volume of roughly 100-200 $\mu \mathrm{l}$ when both nostrils are sprayed. Standard spray pumps will deposit most of the sprayed dose into the anterior region of the nasal cavity. Surface tension of the droplets and mucus layer will cause the immediate spread of the spray. Afterwards mucociliar clearance will distribute the liquid layer within the nasal cavity. Since the nasal mucus layer is continuously renewed and discarded into the throat, the nasal dwelling time of the administered drug depends on how fast it dissolves within the mucus layer and penetrates into the mucosa (Suman et al., 2002).

Although authorities require a lot of data to describe nasal spray devices, for deposition efficiency the plume angle and administration angle are critical factors, while many other spray parameters, including particle size, have relatively minor influences on deposition within the nasal cavity (Foo et al., 2007). These parameters will be discussed in more detail below.

\section{Available technology and requirements}

\subsection{First steps to identify the right spray pump}

Developing a suitable formulation with one or more active ingredients is a lengthy and challenging process. Rarely is a formulation just a simple water-based solution. Auxiliary compounds often have to be added, for example to enhance solubility and stability, to increase viscosity or to prevent microbiological contamination. Once the final formulation is available, the next step is to ensure that the ingredients do not affect the function and integrity of the container closure system (CCS). In the ICH guidelines it reads as follows: The suitability of the container closure system used for the storage, transportation (shipping) and use of the drug product should be discussed. This discussion should consider, e.g., choice of materials, protection from moisture and light, compatibility of the materials of construction with the dosage form (including sorption to container and leaching) safety of materials of construction, and performance (such as reproducibility of the 
dose delivery from the device when presented as part of the drug product (ICH Guideline, 2005). The two topics we want to focus on are compatibility and performance.

To understand the potential problems an insight into the technology of a nasal spray pump system seems beneficial.

The manufacturer fills the drug formulation into multi-dose bottles made of glass or different plastic materials, which are closed by attaching the spray pump including a dip tube. The pump may be fixed by a screw closure, crimped on or simply snapped onto the bottle. Now the system should be tight and no leakage should be observed during subsequent handling. During shipment and storage, the drug formulation is only in contact with the bottle, the outer side of the dip-tube, the gasket between the pump and the bottle and the outer parts of the pump. It is important to recognize, that in this stage the formulation usually does not have contact with inner parts of the pump nor the actuator. So the bottle will provide the largest surface but also the gasket material should be considered when stability issues (e.g. drug sorption, leachables) occur.

Before someone can use the system, the pump must be primed which is normally done by the patient just before first use. A number of priming strokes are required to purge the air off the system and dip tube and to deliver the product at the targeted dose volume. Nasal spray pumps are displacement pumps. When actuating the pump, a piston moves downward in the metering chamber. A valve mechanism at the bottom of the metering chamber will prevent backflow into the dip tube. So the downward movement of the piston will create pressure within the metering chamber which forces the air (before priming) or the liquid outwards through the actuator and generates the spray. When the actuation pressure is removed, a spring will force the piston to return to its initial position. This creates a vacuum in the metering chamber which pulls the liquid from the container by lifting up the ball from the ball seat above the dip tube at the bottom of the metering chamber. It should be recognized, that the "next dose" may be under normal use conditions in contact with the materials for hours or even days when the pump is not regularly used.

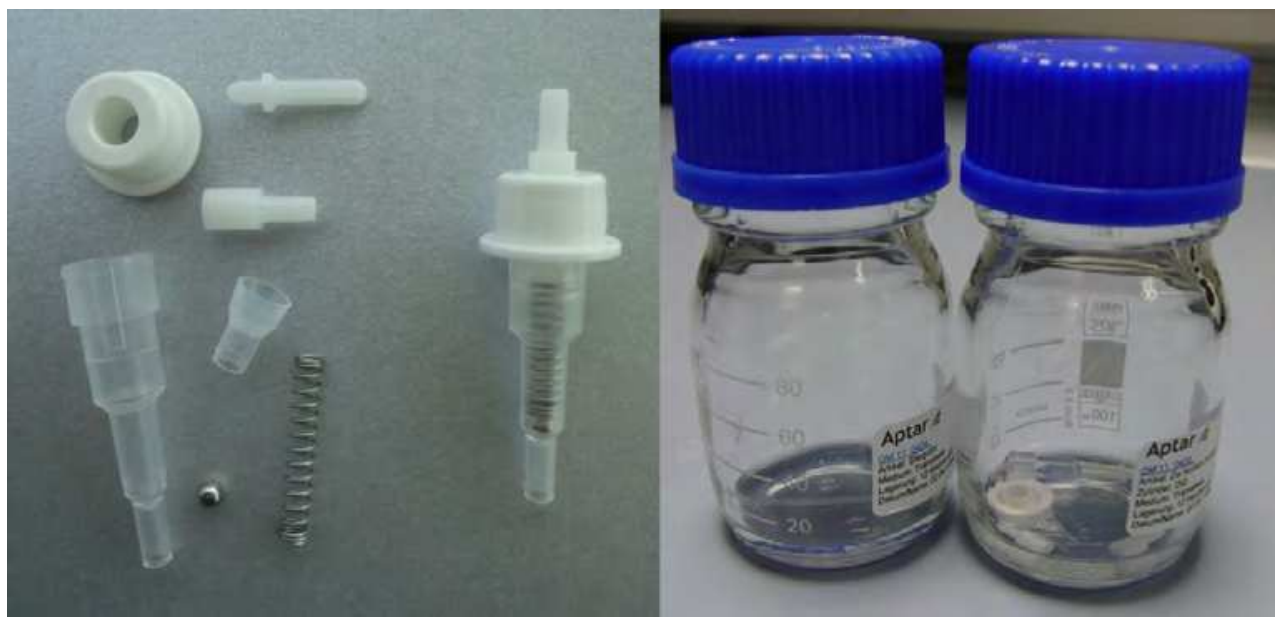

Picture 1. Parts for a standard pump and how it looks like when assembled. On the right side: soaking of parts in formulation to check compatibility. For demonstration just parts for one pump are depicted. 
It is recommended to evaluate effects on proper device function and compatibility of all parts which come in contact with the formulation early in the development process. This can be done in a simple way: disassembled pumps are stored for some days in the drug formulation under controlled conditions. This is done because some ingredients may cause swelling of plastic materials which may result in malfunction of the system. After appropriate storage time the parts are checked for discoloration and critical dimensions. Then the components of the pump system are cleaned and assembled and the proper function of the pump is evaluated. If the pump performs according to the agreed specification further problems are unlikely and the evaluation of performance parameters can start. These simple procedures can detect incompatibilities quite early and it is much better than to find out such issues later in the development stage or even after market launch due to complaints from consumers and patients.

\subsection{General requirements from authorities}

According to EU and FDA guidelines (EMEA guideline, 2006, FDA Guidance for Industry 2002) relevant information should be provided on the characteristics of each of the critical components of the CCS. Critical components are defined as:

- those that come into contact with the patient's mouth or nose or with the formulation

- those that affect the overall performance of the device and

- $\quad$ any additional protective packaging (FDA Guidance for Industry)

Within this chapter we cannot discuss all requirements in detail, but will provide an overview on the most important parameters (also see Table 3). For most parameters you just need to know that they are required and the pump supplier will disclose this information by providing the appropriate files or via referencing a drug master file type III for the US FDA.

\section{Dimensions and used materials}

Dimensions

Material used

\section{Performance}

Shot weight/dose weight

Priming

Droplet size distribution

Spray pattern/plume geometry

Actuation force
Critical dimensions for all individual components and drawings of the device and detailed description of all parts, manufacturing and assembly steps

Documentation on raw material, additives (e.g. colors, stabilizers) processing aids (e.g. lubricants) used for manufacturing including source and quality control

the quantity of drug substance/spray weight expected to be released from the device during actuation EU: mean shot weight/dose within $\pm 15 \%$ of target dose US: mean shot weight/dose within $\pm 10 \%$ of target dose For small doses $(<20 \mu \mathrm{g})$ other criteria may be justified Number of actuations needed to prime/re-prime the pump and obtain the full dose

Description of droplet size distribution and quantification of fine particles (particles $<10 \mu \mathrm{m}$ diameter)

Parameters to describe size, shape and pattern of a delivered spray

Minimum actuation force to achieve desired spray characteristics

Table 3. Key requirements for nasal spray pumps 


\subsection{Overall general description of the device and compatibility issues}

The pump manufacturer needs to provide technical drawings of the assembled device as well as for each individual part with critical dimensions which are controlled during production. Also the manufacturing of each part and the assembling process needs to be described in detail, additional operations like washing or coating steps, sterilization procedures and of course detailed quality control measures.

Since nasal sprays and drops are liquid medications, there is a high likelihood of interactions between the immediate packaging and the formulation. Therefore high quality standards for the used materials must be met. Preferably, only material is used which is described in a Pharmacopea or in foodstuff legislation (EMEA Guideline, 2005). Of course other materials may be also used but in such a case an extractable profile and complete documentation on safety and toxicology needs to be generated and submitted.

So all used materials must be identified including the monomers, any additives or colors used and the suppliers for the raw material must be disclosed. To deal with it, it is important, that the manufacturer of the devices has an established quality and change control process which documents all changes and gives notice to customers when required.

\section{Definitions}

Extractables: compounds which may be extracted from the container closure system by using stressful conditions.

Leachables: compounds which may leach from the container closure system into the formulation under normal conditions of storage and use.

Even when material of high quality is used for the devices some interactions with the formulation can occur.

To ensure proper function of the pump, steel springs and balls are used which may be in contact with the formulation. Any metal components like balls and springs are prone to cause problems. Even if they are made of non-corrosive material, the surface can discolor the formulation due to impurities or contamination with lower grade material during the manufacturing process. Another risk coming from metal parts are remaining lubricants, which can contaminate the product.

Polyoxymethylene (POM), a widely used material for gaskets and pump parts can release formaldehyde into the drug. Materials like polyethylene or polypropylene are less likely to cause problems, but some monomers or additives can leach into the formulation. This problem of leachables coming from the pump is sometimes overlooked, because during the standard stability studies with non-primed pumps no interaction will be seen with parts inside the pumps. When the pumps are tested for dose consistency, the bottle will be emptied within a few hours. So it is recommended to evaluate compatibility of all parts which come in contact with the formulation as described above early in the development process.

\subsection{Performance parameters}

As already mentioned, plume geometry and administration angle have the most significant influence onto nasal deposition but some more parameters are required by authorities for nasal sprays and will be discussed briefly.

It should be clear that the parameters discussed below depend on the experimental conditions and the equipment used for the characterization. So it is important to use well 
controlled conditions (e.g. automated actuation at defined speed or force) and procedures for the measurement of performance parameters. From experience it can be told, that the combination of a particular spray pump with a formulation will result in a quite unique performance profile which is hard to meet when considering the use of another pump. The US-FDA recognized that and recommends for generic nasal sprays to use the same brand and model of devices (particularly the metering valve or pump and the actuator) as used in the reference product to get equivalence on the basis of in vitro tests (FDA Guidance for Industry, 2003).

\subsubsection{Spray pattern and plume geometry}

The spray pattern is best described as a horizontal cut through the spray plume at a defined distance from the orifice, in most cases set at $30 \mathrm{~mm}$. It can be assessed by simply spraying a stained formulation on a sheet of paper or thin layer chromatography (TLC) plates. For TLC plates some chemical reactions may be used to develop the pattern, which is considered more accurately. A higher sophisticated method is the use of a laser beam for the measurements, with systems e.g. from Proveris Scientific, Malvern Instruments, Oxford Lasers or InnovaSystems to name the most widely known providers. Then the shape (e.g. round or ellipsoid shape) and dimensions (e.g. shortest and longest axis) are evaluated and the spray angle may be calculated from this data.

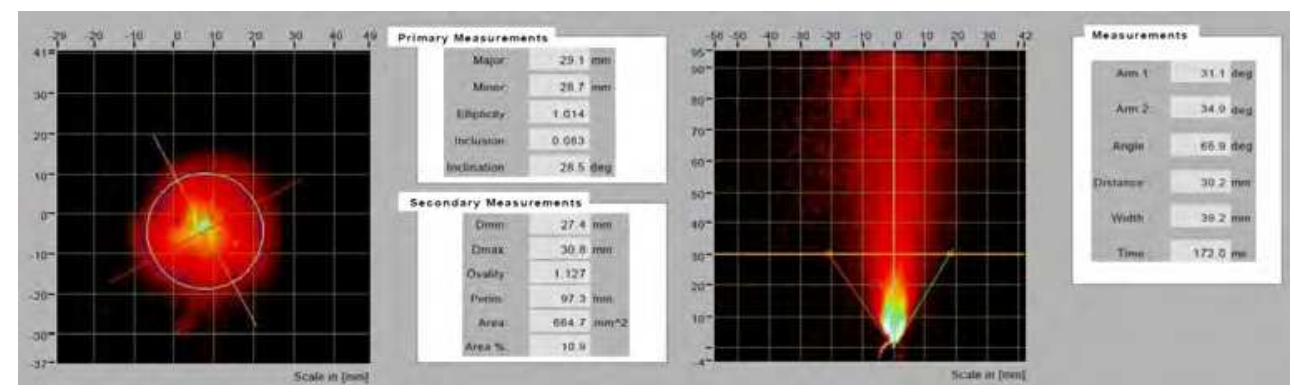

Picture 2. Spray pattern (left) and plume geometry (right) measurements and derived parameters using a laser-based system (Equipment: Proveris Scientific SprayVIEW@ and analysed by Proveris Scientific Viota software).

The plume geometry describes the shape and size of the plume (length, width, spray cone angle) at a defined time point following actuation, preferably during the fully developed phase. These parameters are closely linked to a good nasal deposition but are also important for evaluating the performance of the pump. It is recommended to test a range of pumps for a new formulation, because various factors affect the spray pattern and plume geometry, e.g. the size and shape of the nozzle, the design of the pump, and the size of the metering chamber. On the other side, these parameters are quite sensitive to changes in dimensions within the spray pump as well as changes in the formulation. For these reasons, the spray pattern is often used for quality control purposes.

\subsubsection{Particle-size distribution}

The nose is a very effective filter and most particles and droplets will be caught within the nasal cavity. Only particles less than $10 \mu \mathrm{m}$ median aerodynamic diameter, so called fine 
particles, can reach the lower airways during nasal breathing (Stuart 1984). Most spray pumps will generate an aerosol with a mean particle size in the range of $20-100 \mu \mathrm{m}$ which are recognized as fine mist and will deposit well in the nasal cavity. During the formation and dissipation phases much larger droplets $(>300 \mu \mathrm{m})$ can be formed. Droplet size distribution for nasal sprays is assessed by laser diffraction methods. Results are typically expressed as a size in microns below which $10 \%, 50 \%$ or $90 \%$ of the volume ( $D_{V}$ or mass $D_{M}$ ) of material exist (e.g. $D_{\vee} 10, D_{\vee} 50, D_{\vee} 90$, span) and percentage of droplets less than $10 \mu \mathrm{m}$.

Although a wide range of particles will certainly deposit in the nose, authorities require their characterization because it is a sensitive parameter to detect changes in pump quality or in the formulation.

\subsubsection{Actuation force}

Nasal spray pumps are normally actuated by a fast movement of thumb, index and middle finger. More recently also pumps with side actuation became available. When actuated, the pumps should deliver the whole dose as fine mist within a fraction of a second. Actuation forces should be in the range of 30-80 N ( 3-8 kg). As a rule of thumb, higher viscosity will require higher actuation force. Of course there is also a great impact coming from the device side. Geometry of the metering chamber, design of the valve mechanisms, internal friction, spring forces, dimensions of the swirling chamber have a great influence on this parameter. Some of them can be adapted by the manufacturer to a certain degree to meet the requirements of a particular formulation. Beside this, it is always recommended to test several pump types with a new formulation to find the best match and to meet requirements of the target group (children, elderly).
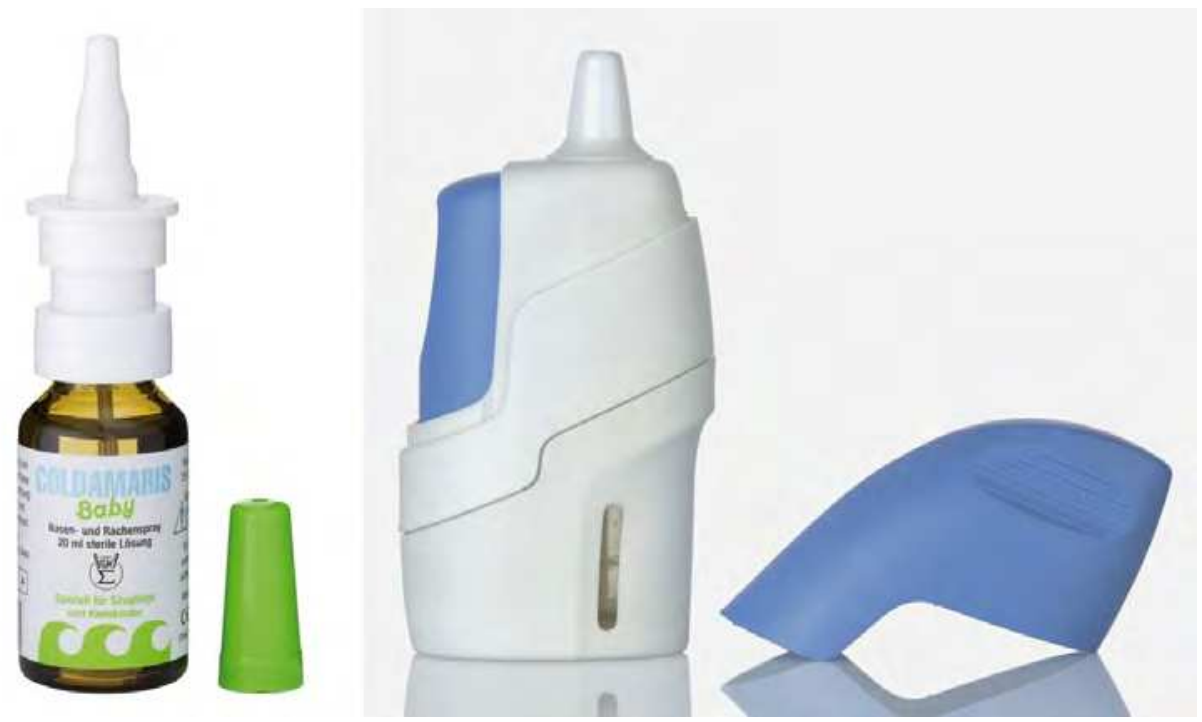

Picture 3. Standard nasal spray pump on the left: This pump is actuated by pressing the actuator in a longitudinal movement towards the bottle. On the right side a nasal spray pump (Latitude ${ }^{\circledR}$ ) designed for lateral actuation with the thumb which is preferred by some people. 


\subsubsection{Shot weight and delivered dose}

Methods for the measurement of shot weight or delivered dose and acceptance criteria are well described in the appropriate guidelines. It should be recognized, that there are some differences between European and US guidelines which should be considered during development.

The volume of the metering chamber will define the delivered dose for a primed pump. This works normally fine for water or saline when the right actuation parameters (force, stroke acceleration) are used. Depending on surface tension and viscosity of the product, air may be trapped in the dosing chamber influencing priming and dose accuracy. Similar problems may occur when air bubbles come into the dip tube. Also too low actuation forces may lead to partial metering. To overcome the problem of partial metering, so called "userindependent" spray pumps were developed but are not widely used by the industry yet.

\section{Bottles}

Bottles or containers are an integral part of drug delivery devices and will also influence the general appearance of the final product. Special shapes may be used to differentiate a product from competitors. Glass bottles are less prone to cause interactions and will give good protection to the formulation even during long storage intervals. Sometimes the glass can influence the stability of the formulation (change in $\mathrm{pH}$, release of trace metals). This depends of course on the quality of the glass which is described by its hydrolytic class (hydrolytic class I-III is normally used for pharmaceutical products). The disadvantages which glass bottles may have are the higher weight and the risk to break when dropped.

Bottles made of plastic material (e.g. polyethylene, polypropylene, PET) are sometimes used for nasal sprays but are mandatory for ophthalmic droppers because squeezing the bottles is needed to dispense the product. Pump supplier will most likely not manufacture these bottles themselves because a complete different technology is used. Parts for spray pumps or droppers are quite exclusively made by injection molding which gives high precision. Plastic bottle manufacturers use a process called blow-molding. The general principle is to make a hollow raw part and then blowing up the material to the final dimensions. The most important disadvantage for all bottles made of plastic material is evaporation/weight loss. Plastic materials are not a perfect barrier for gas or water evaporation. This problem can be tackled using laminated materials but these are more expensive. Another potential risk has to be considered: inks and adhesives from labels may migrate through the bottle wall and leach into the formulation.

Pure mechanics but critical for all types of bottles: the bottle opening must fit the delivery device exactly. It needs to be tested and dimensions need to be controlled because variations may cause leakages or destroy the closure during final assembly. To avoid troubles, consultation of the delivery system supplier is highly recommended as these companies are experienced in managing this interface. The pump supplier should be able to recommend a range of suited bottles from suppliers which provide reliable quality. Before switching to another bottle or bottle supplier, the compatibility with the device should be checked in advance.

\section{Use of preservatives in multi-dose products}

Multi-dose dispensers are widely used for the administration of liquid nasal, ophthalmic or dermal drugs because they are convenient and cost effective. To prevent degradation of the 
product and to avoid the spread of potentially harmful microbes the content has to be kept sterile, both during storage and while in use by the patient or consumer. This requirement is in most cases met by a closely controlled manufacturing and filling procedure and by placing a suitable preservative or a combination of multiple preservatives in the formulations. Benzalkonium chloride (BAC) is by far the most widely used preservative, but thiomersal, chlorhexidine, chlorobutanol and phenylethanol, and parabens can also be found in topical medications (Freeman and Kahook, 2009).

Two general issues are linked to the use of these preservatives, one of which is the choice of materials. This is certainly of primary importance for the manufacturer only. Traditional glass containers do not interact with preservatives, but more and more used plastic containers and dispensing devices pose problems such as permeation of preservatives through the container or interaction with the plastic materials used in these. Rubber also reacts with preservatives but it is still used for closures, which in such events have to be pretreated with the preservatives to minimize subsequent uptake during storage.

The issue of significance for the patient and consumer, however, is the high incidence of local side effects attributed to preservatives. The discussion is controversial, and published preclinical and clinical studies are not always consistent. It seems to be clear that short-term use of preparations containing preservatives at low concentrations is well tolerated, but preservatives can cause serious inflammatory effects with long-term use. The responses may include chemical irritation, hyperreactivity and true allergies (Hong and Bilory, 2009). The German Authorities (BfArM) addressed the use of BAC for nasal sprays in 2003 (BfArM notice, 2003) which pushed the preservative free systems for this administration route. Only recently, European authorities encourage the use of unpreserved multi-dose dispensers even for ophthalmic use (EMEA public statement, 2009).

Systems allowing multi-dose use of unpreserved formulations have the potential to solve some of the problems faced by pharmaceutical manufacturers (e.g. incompatibilities), as well as offer marked benefits for patients in need of chronic treatment. The technology starts to get widely known as PFMD devices (preservative free multi dose).

When using a preserved or unpreserved multi-dose product, there are three ways for microorganisms to enter the system:

- $\quad$ via the orifice and

- $\quad$ via the venting air which is replacing the dispensed liquid or

- insufficient container/dispenser fit.

In preserved formulations (conventional system), the added preservative controls microbial growth and no additional measures need to be taken to prevent microbial occupation via the orifice or venting air. If the formulation does not contain preservatives, the device must be able to keep microorganisms out off the system.

Today a range of technical solutions is available to overcome this issue. The highest risk of contamination obviously comes from the orifice, because it may come in contact with skin and mucosa as well as with infected body fluids. Some marketed systems use the oligodynamic activity of a silver wire in the tip of the actuator, a silver coated spring and ball (Groß, 2000). These components release silver ions into the formulation, which is a time dependent process. The system is able to keep microorganisms down between long dosing intervals, even when the tip is immersed into bacterial contaminated fluid (Bagel and Wiedemann, 2004). Silver ions are widely used for their antiseptic properties and even when used for wound dressings, it is safe and no adverse effects are attributed to this treatment. One general limitation of course must be considered: the silver ions may react with the 
formulation, e.g. chloride ions and form micro-precipitations. This effect may be overlooked because it is most relevant for spans of 6-12 hours between individual actuations which are not routinely tested during development.
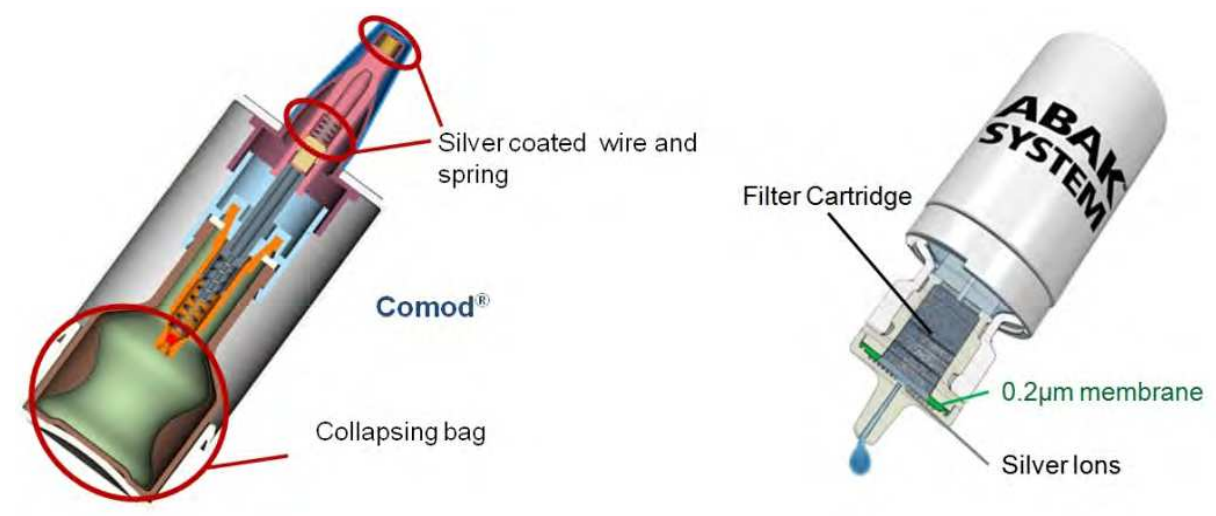

Picture 4. Examples for ophthalmic devices using the oligodynamic effects of silver in combination with other measures like collapsing bags or sterile filtration of the venting air.

Consequently, the most recent preservative free systems follow a purely mechanical approach to minimize interactions between parts of the device and the formulation. One technical solution to prevent contamination via the orifice is named "tip seal technology" which may be utilized for both nasal spray pumps and ophthalmic droppers. A spring loaded valve is located directly below the opening of the tip orifice and does not allow any microbes to migrate from any surfaces or contacted liquids into the system, the orifice is "sealed" under resting conditions. The tip seal keeps the system closed until a defined pressure (for nasal sprays it is more than 3 bar) is reached by actuating the system. Then the system will open and the formulation is forced through the orifice with a higher pressure than needed to open the valve. When the pressure drops at the end of the actuation the tip seal will immediately close the orifice with an outward movement. So no backflow of potentially contaminated medication or other liquid is possible. Depending on the pump system, the fluid path may even be "metal-free", which means the springs needed for the device operation do not come in contact with the formulation.

To avoid contamination of the formulation via venting air different technical solutions are used. The simplest way is sterile filtration of the venting air using separate filters or filter gaskets. For oxygen-sensitive formulations, so called collapsing bags or depressed systems are used. The formulation is filled in a special, microbial tight bag which is protected by a surrounding bottle. When dispensing the product, the bag collapses with the content not coming in contact with the ambient air. Some pumps are constructed in such a way, that the whole system is air-thigh and during use a certain vacuum (up to -300 mbar) is generated within the bottle. Those systems allow even a purging with inert gases to reduce oxygen content in the container head space.

These approaches to avoid the use of preservatives for multi-dose devices sound complicated but are well established and mature technologies. Preservative-free single dose 
containers, most often presented as blow-fill-seal (BFS) containers can be an alternative for short term treatments. Disadvantages of these systems are linked to the quite complicated filling technology, the need to overfill and amount of material needed for each dose. Any efforts to provide reliable preservative free multi-dose systems are certainly appreciated by the growing number of patients who experienced discomfort with preserved formulations.

\section{Prevent contamination via orifice}

Oligodynamic components in the actuator or inside the pump

"tip-seal" technology

Prevent contamination via venting air Sterile filter incorporated into delivery system

Collapsing primary container

Unvented container/pump system
Open orifice, but silver-ions are released into the formulation which kills germs but may interact with the formulation

Mechanical barrier system to prevent bacterial contamination

The venting air in pressure balanced systems are forced though sterile filters with pore sizes less than $0.2 \mu \mathrm{m}$, the filter membranes are normally hydrophobic which prevents leakage from liquids out of the container via the venting system Best suited for oxygen-sensitive formulations, because the venting air does not come in contact with it, this technology also enables spraying at different angles

Tight fit of bottle and pump design prevent venting of the bottle, vacuum up to -300 mbar are reached

Table 4. Measures/technical solutions for preservative free multi-dose devices

\subsection{Preservative free ophthalmic devices}

With aging populations, conditions which require chronic treatment like dry eye syndrome or glaucoma are on the rise (WHO fact sheet, 2009) and preservative free multi-dose devices come into play. Chronic use of preserved formulation is not always tolerated by some patients. That is why the EMA encourages the development of such devices (EMEA public statement, 2009). This is not an easy task and poses some risks for the manufacturer. The standards for ophthalmic medications are set high to make sure that no patient or consumer 'risks an eye'. Agencies enforce guidelines on sterility of the product, absence of particles and microbial stability. Typically, the observation of microbial growth in a product leads to rejection of the entire batch, which will impact the manufacturer significantly.

Production and filling of ophthalmic medications are highly sophisticated, so pharmaceutical manufacturers are reluctant to alter established processes or to invest substantially in new filling technologies that obviously require further process qualification.

Because of these difficulties, only a very few multi-dose devices are available on the market yet, most of them relying on the oligodynamic effects of silver ions.

A patient-friendly, multi-dose system for unpreserved liquids is what patients and consumers are looking for, regardless whether one considers "simple" products like artificial tears or more complex pharmaceutical products such as prostaglandins or the like for treatment of serious diseases such as glaucoma. 

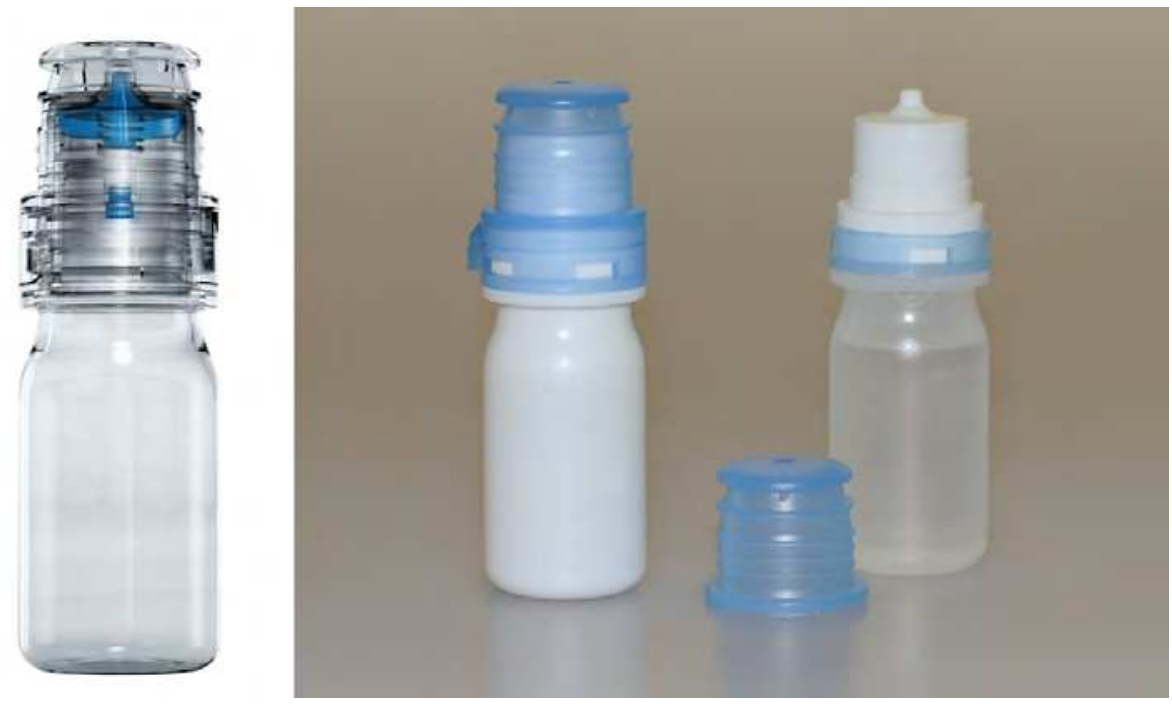

Picture 5. On the left: Rendered picture of the Ophthalmic Squeeze Dispenser (OSD) showing the tip seal and filter for the venting air in blue. Right: the complete system after filling and with removed protection cap.

A break through approach may be the Ophthalmic Squeeze Dispenser (OSD) which uses the mechanical tip-seal technology combined with sterile filtration of the venting air known from preservative free nasal spray pumps. The OSD is simply actuated by squeezing a LDPE (low density polyethylene) bottle and will deliver a drop in the range of $35 \mu$. The device can easily be adapted for a certain range of viscosity to keep forces within a rational limit and to deliver a consistent drop. Not only is the dropper highly sophisticated but the bottle too. The thickness of the bottle wall must be a compromise between minimizing water vapor loss and providing acceptable actuation forces. Of course the fit between bottle and dropper must be perfect to avoid contamination via this way. As some ophthalmic formulations have compatibility issues with metal, the OSD features a metal-free fluid path.

\section{Microbial device integrity tests}

To introduce a preservative-free multi-dose device successfully on the market, the microbial integrity must be clearly demonstrated to customers and authorities. Although there are guidelines in place for preserved multi-dose and preservative-free single dose containers, there is no guideline on microbial testing of preservative-free multi-dose devices. So the industry has to develop convincing test methods on their own. A well known procedure is the so called Wiedemann test, which was developed to characterize the 3K/Comod-system (Bagel and Wiedemann, 2004). This test for nasal sprays focuses on the microbial load of the next sprayed dose and was developed according to the needs of the system which releases silver ions into the formulation. The acceptance criteria are less than $100 \mathrm{CfU} / \mathrm{ml}$ delivered dose (according the EU Pharmacopeia) and no contamination of the bottle content. Similar tests were developed using a very agile germ (Pseudomonas aeruginosa) for the tip seal test and the tiny and robust spores from Baccilus subtilis for the whole package integrity test. These tests may be used for the characterization of preservative-free multi-dose nasal spray 
devices (Bommer et al., 2004) as well as for ophthalmic devices (Marx et al., 2010). For such device qualification tests most often the devices are filled with broth medium provide optimum conditions for the germs to growth in the case of any contamination. The final product will promote bacterial growth much less which provides more confidence.

\section{TIP SEAL INTEGRITY TEST (TSIT) FOR DEVICE QUALIFICATION}

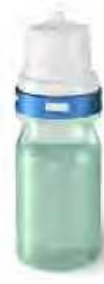

Fill container with sterile peptone water, mount sterilized dropper, and check microbial status.

Microbial status of delivered doses Before starting the challenge, 4 hours after first (day 1), at day 3 prior to challenge and at day 8 the microbial load of the delivered drop is analyzed.

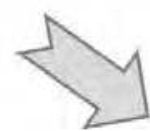

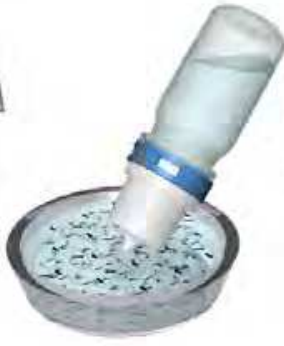

Incubate after last challenge for 3 days at $36^{\circ} \mathrm{C}$. Check microbial contamination of bottle content Clear medium $=$ no bacterial ingress
Prepare challenge suspension containing $10^{7} \mathrm{CfL}$ Pseudomonas aeruginosa (ATCC 9027). Actuate dropper with immersed tip-seal 2 times daily for 5 days, incubate in-between at $36^{\circ} \mathrm{C}$.
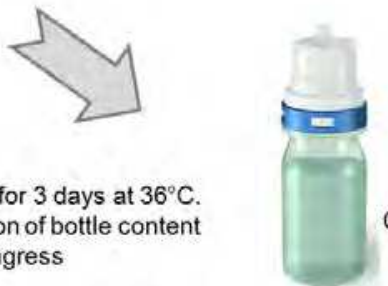

Clear medium

Picture 6. Microbial test to challenge the tip seal function and contamination of delivered doses with a preservative-free ophthalmic dropper.

\section{Summary}

The intranasal route can be used for a wide range of applications and administration procedure is safe and easy. On the other side, development of a nasal formulation and to identify the right device is a complex process. Cooperation with an experienced pump manufacturer and specialized test laboratories on the selection of the delivery system and setting the right specifications can save resources and increase probability of success. Selecting the wrong spray pump or bottle can jeopardize the entire project so a range of pumps should be tested early in the development process. In this chapter we have focused on multi-dose devices for liquids because they clearly dominate the market. In the future we will see more of so called unit-dose devices which deliver only one dose for one or both nostrils and are discarded then. Such devices can be used for the administration of controlled substances (like fentanyl) to reduce the risk of misuse or for vaccinations (Marx et al., 2011). There are also some propellant-driven devices on the market (e.g. for nasal steroids) but discussion about the impact on the environment may limit their success. The future will also see powders for intranasal administration but all known projects are still in various stages of clinical trials. Last not least electronic add-ons, such as counters or locking features, may bring advantages and opportunities in nasal drug delivery, but will obviously need to comply with cost challenges. 


\section{CLOSURE AND VENTILATION INTEGRITY TEST (CVIT) FOR DEVICE QUALIFICATION}

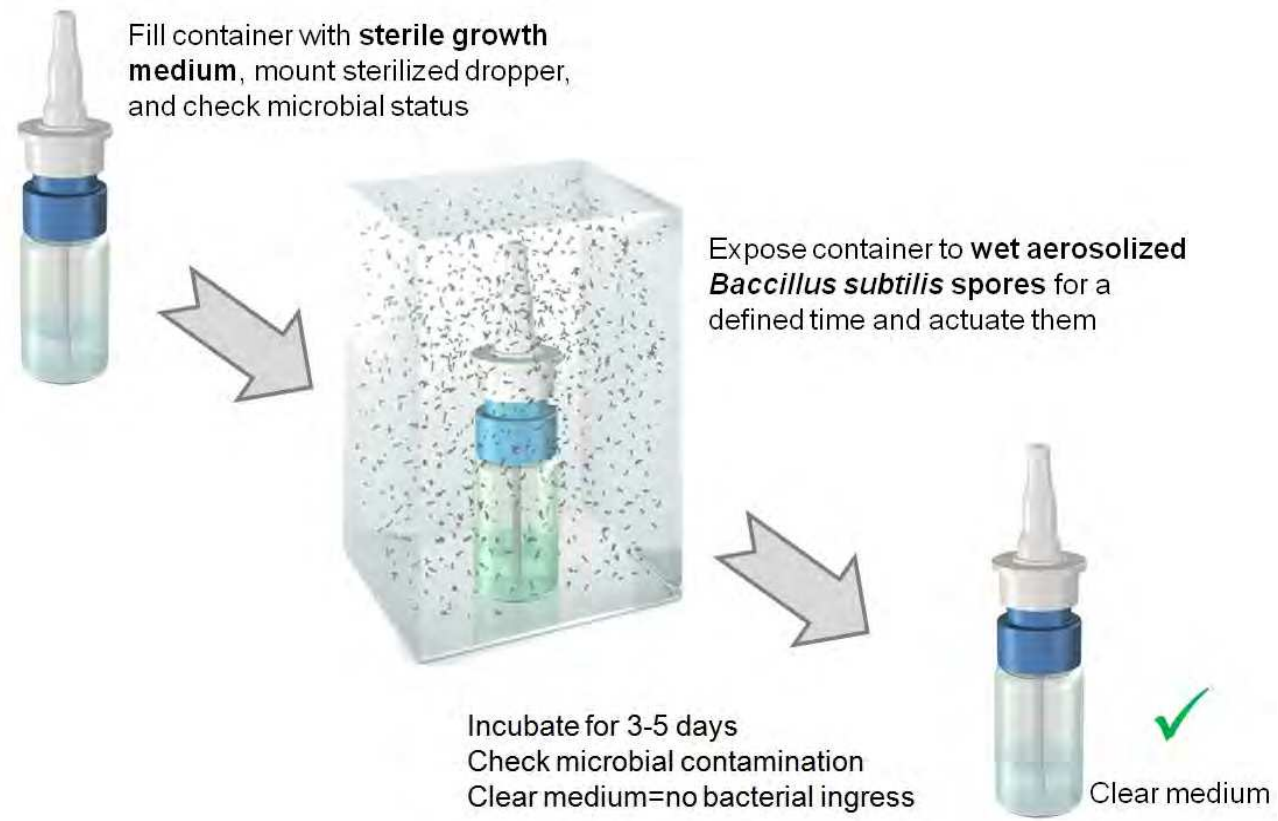

Picture 7. Microbial test to challenge the venting system and closure tightness for preservative free multi-dose systems. During the aerosol-exposure the systems are actuated to generate an under pressure within the system.

Non-invasive administration of eye care products is still by far the most used way to reach therapeutic targets, let it be in diseases such as glaucoma or in self-medicated conditions such as dry-eye syndrome. Alternative methods such as implants or intravitreal injections are getting more attention lately, but given the implications associated with these methods it is fair to conclude that the multidose eye dropper still has a promising future. Avoiding or at least reducing the use of preserving agents in ophthalmic formulations is one way to maintain or extend life cycles of existing products and to ease acceptance of new entities. However, in particular in eye care habits of patients and consumers need to be taken into account carefully.

To answer the question from the beginning: will drug formulations for multiple use, presented in multi-dose containers for nasal or ophthalmic drugs, become preservative free? The answer is not as easy to give as it seems, because cultural aspects are no less important than regulatory aspects, safety concerns or clinical findings. However, as seen today in Europe, a trend begins to establish itself towards preservative-free multi-dose formulations and, consequently, delivery systems, in order to meet patient's expectations. Any development of novel drugs or research on life cycle management should take this into serious consideration. 


\section{References}

Bagel S, Wiedemann B. Extension of in-use stability of preservative-free nasalia. Europ J Pharmaceutics and Biopharmaceutics. 2004, (57): 353-358

Bommer R, Kern J, Hennes K, Zwisler W. Preservative-free nasal drug-delivery systems. Med Dev Technol. 2004 (10): 2-5

Bescheid des Bundesinstitut für Arzneimittel und Medizinproduke für benzalkoniumchlorid-haltige Arzneimittel zur Anwendung in der Nase, A 37489/38186/03 Bonn, Dezember 2003

Committee for medicinal products for human use (CHMP). Guideline on the pharmaceutical quality of inhalation and nasal products. London: European Medicines Agency; 2006

EMEA Public statement on antimicrobial preservatives in ophthalmic preparations for human use. London: European Medicines Agency, Dezember 2009, Doc-Ref. EMEA/622721/2009

Guideline on plastic immediate packaging Materials. London: European Medicines Agency; May 2005

Guidance for Industry: Nasal Spray and Inhalation Solution, Suspension, and Spray Drug Products - Chemistry, Manufacturing, and Controls Documentation. US Department of Health and Human Services, Food and Drug Administration, Center for Drug Evaluation and Research (CDER). Rockville, MD; 2002

Guidance for Industry (draft guidance): Bioavailability and Bio-equivalence Studies for Nasal Aerosols and Nasal Sprays for Local Action, US FDA April 2003

Foo MY, Cheng YS, Su WC, Donovan MD. The influence of spray properties on intranasal deposition. J Aerosol Med. 2007, 20(4): 495-508

Freeman DP, Kahook MY. Preservatives in topical ophthalmic medications: historical and clinical perspectives. Expert Rev Ophthalmol. 2009; 4(1): 59-64

Groß D. The COMOD-System - a preservative free drug therapy against glaucoma. 321-328, in Orgül/Flammer (Editors): Pharmacotherapie in glaucoma, Bern 2000

Hong J, Bielory L. Allergy to ophthalmic preservatives. Curr Opin Allergy Clin Immunol. 2009;9(5):447-453.

ICH Harmonised Tripartite Guideline, Pharmaceutical Development Q8; Step 4 version, November 2005

Leary AC, Dowling M, Cussen K, O'Brien J, Stote RM. Pharmacokinetics and pharmacodynamics of intranasal insulin spray (Nasulin) administered to healthy male volunteers: infuence of the nasal cycle. J Diabetes Sci Technol. 2008, 2(6): 1054-60

Marx D and Birkhoff M. New Devices for Dispensing Ophthalmic Treatments May Be the Key to Managing the Life Cycles of Established Products. Drug Delivery Technology 2010, 10 (9): 16-21

Marx D, Leitz M, Fagot C. Do We Need New Devices for Intranasal Vaccination? Drug Development \& Delivery. 2011, 11 (3): 54-59

Stuart BO. Deposition and clearance of inhaled particles. Environ Health Perspect. 1984; 55: 369-390

Suman JD, Laube BL, Lin TC, Brouet G, Dalby R. Validity of in vitro tests on aqueous spray pumps as surrogates for nasal deposition. Pharm Res. 2002;19(1): 1-6

Wen MM. Olfactory targeting through intranasal delivery of biopharmaceutical drugs to the brain - current development. Discov Med. 2011 Jun;11(61): 497-503

WHO Fact Sheet No. 282. Visual impairment and blindness. May 2009 


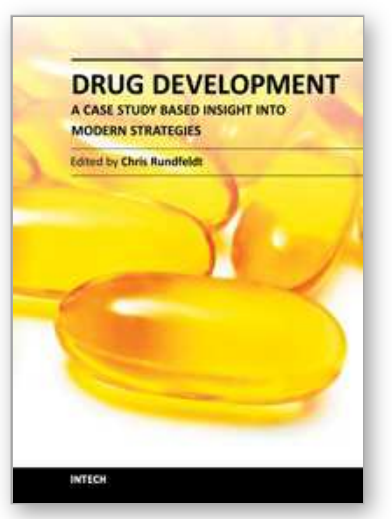

\author{
Drug Development - A Case Study Based Insight into Modern \\ Strategies \\ Edited by Dr. Chris Rundfeldt
}

ISBN 978-953-307-257-9

Hard cover, 654 pages

Publisher InTech

Published online 07, December, 2011

Published in print edition December, 2011

This book represents a case study based overview of many different aspects of drug development, ranging from target identification and characterization to chemical optimization for efficacy and safety, as well as bioproduction of natural products utilizing for example lichen. In the last section, special aspects of the formal drug development process are discussed. Since drug development is a highly complex multidisciplinary process, case studies are an excellent tool to obtain insight in this field. While each chapter gives specific insight and may be read as an independent source of information, the whole book represents a unique collection of different facets giving insight in the complexity of drug development.

\title{
How to reference
}

In order to correctly reference this scholarly work, feel free to copy and paste the following:

Degenhard Marx and Matthias Birkhoff (2011). Multi-Dose Container for Nasal and Ophthalmic Drugs: A Preservative Free Future?, Drug Development - A Case Study Based Insight into Modern Strategies, Dr. Chris Rundfeldt (Ed.), ISBN: 978-953-307-257-9, InTech, Available from: http://www.intechopen.com/books/drugdevelopment-a-case-study-based-insight-into-modern-strategies/multi-dose-container-for-nasal-andophthalmic-drugs-a-preservative-free-future-

\section{INTECH}

open science | open minds

\section{InTech Europe}

University Campus STeP Ri

Slavka Krautzeka 83/A

51000 Rijeka, Croatia

Phone: +385 (51) 770447

Fax: +385 (51) 686166

www.intechopen.com

\section{InTech China}

Unit 405, Office Block, Hotel Equatorial Shanghai

No.65, Yan An Road (West), Shanghai, 200040, China

中国上海市延安西路65号上海国际贵都大饭店办公楼405单元

Phone: $+86-21-62489820$

Fax: $+86-21-62489821$ 
(C) 2011 The Author(s). Licensee IntechOpen. This is an open access article distributed under the terms of the Creative Commons Attribution 3.0 License, which permits unrestricted use, distribution, and reproduction in any medium, provided the original work is properly cited. 